\title{
On the volume of the shrinking branching Brownian sausage
}

\author{
Mehmet Öz*
}

\begin{abstract}
The branching Brownian sausage in $\mathbb{R}^{d}$ was defined in [4] similarly to the classical Wiener sausage, as the random subset of $\mathbb{R}^{d}$ scooped out by moving balls of fixed radius with centers following the trajectories of the particles of a branching Brownian motion (BBM). We consider a $d$-dimensional dyadic BBM, and study the large-time asymptotic behavior of the volume of the associated branching Brownian sausage (BBM-sausage) with radius exponentially shrinking in time. Using a previous result on the density of the support of BBM, and some well-known results on the classical Wiener sausage and Brownian hitting probabilities, we obtain almost sure limit theorems as time tends to infinity on the volume of the shrinking BBM-sausage in all dimensions.
\end{abstract}

Keywords: branching Brownian motion; density; sausage; strong law of large numbers.

AMS MSC 2010: 60J80; 60F15; 60D05; 92D25.

Submitted to ECP on December 6, 2019, final version accepted on May 3, 2020.

Supersedes arXiv: 1911.10919.

\section{Introduction}

\subsection{Formulation of the problem and background}

Let $X=(X(t))_{t \geq 0}$ be a standard $d$-dimensional Brownian motion (BM) starting at the origin. The Wiener sausage of radius $r$ associated to $X$ is the set-valued process defined by

$$
X_{t}^{r}=\bigcup_{0 \leq s \leq t} B(X(s), r),
$$

where $B(x, r)$ is the closed ball of radius $r>0$ centered at $x \in \mathbb{R}^{d}$. For each $t \geq 0, X_{t}^{r}$ is then a random subset of $\mathbb{R}^{d}$, which looks like a 'sausage' scooped out over the period $[0, t]$ by a moving ball of fixed radius centered at the Brownian trajectory. Note that the Wiener sausage is a non-Markovian functional of $X$.

Now let $Z=(Z(t))_{t \geq 0}$ be a $d$-dimensional strictly dyadic branching Brownian motion (BBM). The process can be described as follows. It starts with a single particle at the origin, which performs a BM in $\mathbb{R}^{d}$ for a random lifetime, at the end of which it dies and simultaneously gives birth to two offspring. Similarly, starting from the position where their parent dies, each offspring particle repeats the same procedure as their parent independently of each other and of the parent, and the process evolves through time in this way. All particle lifetimes are exponentially distributed with constant parameter $\beta>0$, which is called the branching rate. For each $t \geq 0, Z(t)$ can be viewed as a finite

\footnotetext{
${ }^{*}$ Department of Natural and Mathematical Sciences, Faculty of Engineering, Özyeğin University, Istanbul, Turkey. E-mail: mehmet.oz@ozyegin.edu.tr https://faculty.ozyegin.edu.tr/mehmetoz/
} 
discrete measure on $\mathbb{R}^{d}$, which is supported at the particle positions at that time. We use $P$ and $E$, respectively, as the probability and corresponding expectation for $Z$. The range (accumulated support) of $Z$ is the process defined by

$$
R(t)=\bigcup_{0 \leq s \leq t} \operatorname{supp}(Z(s))
$$

and the branching Brownian sausage (BBM-sausage) with radius $r$ associated to $Z$ is the process defined by

$$
\widehat{Z}_{t}^{r}:=\bigcup_{x \in R(t)} B(x, r)
$$

The Wiener sausage and various set functions of it, especially its volume, have been frequently studied going back to [13]. In [3], Donsker and Varadhan obtained an asymptotic result on the Laplace transform of the volume of the Wiener sausage, which is a large-deviation (LD) result giving information on the probability that the volume is aytpically small. In [1] and [14], the work in [3] was extended to the case of the so-called shrinking Wiener sausage. We refer the reader to [5, Sect. 1] and the references therein for a brief survey of limit theorems on the volume of the Wiener sausage.

The branching Brownian sausage was introduced by Engländer in [4] in analogy with the classical Wiener sausage, and an asymptotic result on the Laplace transform of its volume was obtained similar to the one in [3], by using an equivalence to a trapping problem of BBM among Poissonian traps. In more detail, consider a Poisson point process $\Pi$ on $\mathbb{R}^{d}$ with intensity measure $\nu$, and for $r>0$ define the random trap field as

$$
K:=\bigcup_{x_{i} \in \operatorname{supp} \Pi} B\left(x_{i}, r\right) .
$$

Define the first trapping time of the BBM as $T:=\inf \{t \geq 0: R(t) \cap K \neq \emptyset\}$, and the event of survival of BBM from traps up to time $t$ as $S_{t}:=\{T>t\}$. Then, denoting the annealed law of the traps and the BBM as $\mathbb{P}$, the first trapping problem of BBM among a Poissonian field of traps in $\mathbb{R}^{d}$ is related to the BBM-sausage by Fubini's theorem:

$$
\mathbb{P}\left(\mathrm{S}_{\mathrm{t}}\right)=E\left[e^{-\nu\left(\widehat{Z}_{t}^{r}\right)}\right] .
$$

For a Borel set $A \subseteq \mathbb{R}^{d}$, we say volume of $A$ to refer to its Lebesgue measure, which we denote by $\operatorname{vol}(A)$. In [4], it was shown that when the trap intensity is uniform, that is, when $\nu=v$ vol with $v>0$, then

$$
E e^{-v \operatorname{vol}\left(\widehat{Z}_{t}^{r}\right)}=e^{-\beta t+o(t)}, \quad t \rightarrow \infty .
$$

To the best of our knowledge, apart from [4], no further work was done on the BBMsausage. We note that (1.2) gives information on the probability that the volume of a BBM-sausage with a constant radius is atypically small, whereas in the current work we study the typical behavior of a BBM-sausage with an exponentially shrinking radius.

\subsection{Motivation}

The current work can be regarded as a sequel to the recent works [10] and [11] under the common theme of spatial distribution of mass in BBM. In [10], the mass of BBM falling in linearly moving balls of fixed radius was studied, and an LD result on the large-time probability that this mass is atypically small on an exponential scale was obtained. The asymptotics of the probability of absence of BBM in linearly moving balls of fixed radius, emerged as a special case [10, Corollary 2]. It is well-known that the total mass of BBM typically grows exponentially in time. Also, it is known that typically for large $t$ and any $\varepsilon>0$, at time $t$ there will be particles outside $B(0, \sqrt{2 \beta}(1-\varepsilon) t)$ but no particles outside $B(0, \sqrt{2 \beta}(1+\varepsilon) t)$. 
Definition 1.1 (Subcritical ball). We call $B=\left(B\left(0, \rho_{t}\right)\right)_{t>0}$ a subcritical ball if there exists $0<\varepsilon<1$ and time $t_{0}$ such that $B\left(0, \rho_{t}\right) \subseteq B(0, \sqrt{2 \beta}(1-\varepsilon) t)$ for all $t \geq t_{0}$.

Remark 1.2. We use the term subcritical ball both in the sense of an expanding ball $B=\left(B\left(0, \rho_{t}\right)\right)_{t \geq 0}$ as in Definition 1.1, and also simply as a snapshot taken of an expanding ball at a fixed large time $t$ as $B\left(0, \rho_{t}\right)$.

In [11], the following was asked: how homogeneously are the exponentially many particles at time $t$ spread out over a subcritical ball? This homogeneity question was formulated in terms of the degree of density of support of BBM at time $t$. Firstly, [10, Corollary 2] was extended to the case of the mass falling in linearly moving balls of exponentially shrinking radius $r(t)=r_{0} e^{-k t}$, and then via a covering by sufficiently many of such balls, an LD result concerning the $r(t)$-density of the support of BBM in subcritical balls was obtained. The concept of $r(t)$-density of the support of BBM naturally led to the following definition.

Definition 1.3 (Enlargement of BBM). Let $Z=(Z(t))_{t \geq 0}$ be a BBM. For $t \geq 0$, we define the $r$-enlargement of $Z(t)$ as

$$
Z_{t}^{r}:=\bigcup_{x \in \operatorname{supp}(Z(t))} B(x, r) .
$$

In [11], furthermore, the following results were obtained on the large-time behavior of $r(t)$-enlargement of BBM in $\mathbb{R}^{d}$. Theorem A below says that, with probability one, an $r(t)$-enlargement of BBM with $r(t)$ decaying exponentially in $t$ covers the subcritical ball $B(0, \theta \sqrt{2 \beta} t)$ eventually provided that $\theta$ is smaller than a certain critical value. Theorem $\mathrm{B}$ is on the large-time behavior of the volume of $r(t)$-enlargement of BBM.

Theorem A (Almost sure density of BBM; [11]). Let $0<\theta<1,0 \leq k<\left(1-\theta^{2}\right) / d, r_{0}>0$ and $r: \mathbb{R}_{+} \rightarrow \mathbb{R}_{+}$be defined by $r(t)=r_{0} e^{-\beta k t}$. For $t>0$ define $\rho_{t}:=\theta \sqrt{2 \beta} t$. Then, in any $d \geq 1$,

$$
P\left(\Omega_{0}\right)=1 \text {, where } \Omega_{0}:=\left\{\omega: \exists t_{0}=t_{0}(\omega) \text { such that } \forall t \geq t_{0}, B\left(0, \rho_{t}\right) \subseteq Z_{t}^{r_{t}}(\omega)\right\} .
$$

In what follows, we use $\omega_{d}$ to denote the volume of the $d$-dimensional unit ball.

Theorem B (Almost sure volume of enlargement of BBM; [11]). Let $0 \leq k \leq 1 / d, r_{0}>0$ and $r: \mathbb{R}_{+} \rightarrow \mathbb{R}_{+}$be defined by $r(t)=r_{0} e^{-\beta k t}$. Then, with probability one,

$$
\lim _{t \rightarrow \infty} \frac{\operatorname{vol}\left(Z_{t}^{r_{t}}\right)}{t^{d}}=[2 \beta(1-k d)]^{d / 2} \omega_{d}
$$

Motivated by the results above, we ask the following question in the present work. For large $t$, by how much on the scale of $t^{d}$, if at all, is the volume of the BBM-sausage with radius $r(t)$ (i.e., the $r(t)$-shrinking BBM-sausage) larger than that of the $r(t)$-enlargement of BBM? The aim here is to answer this question in a precise way as $t \rightarrow \infty$.

Notation: We introduce further notation for the rest of the manuscript. For $x \in \mathbb{R}^{d}$, we use $|x|$ to denote its Euclidean norm. We use $c, c_{0}, c_{1}, \ldots$ as generic positive constants, whose values may change from line to line. If we wish to emphasize the dependence of $c$ on a parameter $p$, then we write $c_{p}$ or $c(p)$. We use $\mathbb{R}_{+}$to denote the set of nonnegative real numbers, and write $o(f(t))$ to refer to $g(t)$, where $g: \mathbb{R}_{+} \rightarrow \mathbb{R}_{+}$is a generic function satisfying $g(t) / f(t) \rightarrow 0$ as $t \rightarrow \infty$, unless otherwise stated. Also, for a function $g: \mathbb{R}_{+} \rightarrow \mathbb{R}_{+}$, we use $g_{t}=g(t)$ for notational convenience. We denote by $X=(X(t))_{t \geq 0}$ a generic standard BM in $d$-dimensions, and use $\mathbf{P}_{x}$ and $\mathbf{E}_{x}$, respectively, as the law of $X$ started at position $x \in \mathbb{R}^{d}$, and the corresponding expectation.

Outline: The rest of the paper is organized as follows. In Section 2, we present our main results. In Section 3, we develop the preparation for the proofs of our main results, and then give the heuristic argument behind them. The proofs of the main results are given in Section 4. 


\section{Results}

Theorem 2.1 and Theorem 2.2 are on the almost sure growth of exponentially shrinking BBM-sausages in $d=2$ and $d \geq 3$, respectively.

Let $k \geq 0, r_{0}>0$, and $r: \mathbb{R}_{+} \rightarrow \mathbb{R}_{+}$be defined by $r(t)=r_{0} e^{-\beta k t}$.

Theorem 2.1. In $d=2$, with probability one,

$$
\lim _{t \rightarrow \infty} \frac{\operatorname{vol}\left(\widehat{Z}_{t}^{r_{t}}\right)}{t^{2}}=2 \pi \beta
$$

Theorem 2.2. In $d \geq 3$, with probability one,

$$
\lim _{t \rightarrow \infty} \frac{\operatorname{vol}\left(\widehat{Z}_{t}^{r_{t}}\right)}{t^{d}}= \begin{cases}{[2 \beta(1-k(d-2))]^{d / 2} \omega_{d}} & \text { if } k<1 /(d-2) \\ 0 & \text { if } k \geq 1 /(d-2)\end{cases}
$$

Remark 2.3. Theorem 2.1 says that in $d=2$, the large-time behavior of the volume of $\widehat{Z}_{t}^{r_{t}}$ is as different as it can be from that of $Z_{t}^{r_{t}}$, which is given by Theorem B as: with probability one,

$$
\lim _{t \rightarrow \infty} \frac{\operatorname{vol}\left(Z_{t}^{r_{t}}\right)}{t^{2}}= \begin{cases}2 \pi \beta(1-2 k) & \text { if } k<1 / 2 \\ 0 & \text { if } k \geq 1 / 2\end{cases}
$$

This can be explained as follows. In $d=2$, the motion component of BBM plays a dominating role in the large-time behavior of the shrinking BBM sausage due to the almost sure neighborhood recurrence of BM. Note that the result does not depend on $k$. For large $t$, a BBM-sausage with any exponentially shrinking radius (independent of how large the exponential rate of decay is for the radius) covers all subcritical balls, that is, for any $0<\varepsilon<1$, the sausage $\widehat{Z}_{t}^{r_{t}}$ eventually covers $B(0, \sqrt{2 \beta}(1-\varepsilon) t)$ almost surely.

In $d \geq 3$, from Theorem $\mathrm{B}$, we have

$$
\lim _{t \rightarrow \infty} \frac{\operatorname{vol}\left(Z_{t}^{r_{t}}\right)}{t^{d}}= \begin{cases}{[2 \beta(1-k d)]^{d / 2} \omega_{d}} & \text { if } k \leq 1 / d \\ 0 & \text { if } k>1 / d\end{cases}
$$

Hence, Theorem 2.2 says that in $d \geq 3$ for large $t$, provided that the decay of the sausage radius is slow enough, the accumulated support of BBM over $[0, t)$ has a nontrivial contribution to the volume of the $r_{t}$-shrinking sausage over $[0, t]$ although the contribution is not significant enough to cover all subcritical balls; whereas, if the decay of $r_{t}$ is sharper than a certain threshold (i.e., if $k>1 /(d-2)$ ), the accumulated support over $[0, t)$ and the support at time $t$ both have negligible contribution on the scale of $t^{d}$.

Remark 2.4. In $d=1$, it is clear that with probability one,

$$
\lim _{t \rightarrow \infty} \frac{\operatorname{vol}\left(\widehat{Z}_{t}^{r_{t}}\right)}{t}=\lim _{t \rightarrow \infty} \frac{\left[2 r_{t}+\sup _{0 \leq s \leq t} R(t)-\inf _{0 \leq s \leq t} R(t)\right]}{t}=2 \sqrt{2 \beta},
$$

which follows from the well-known result of Bramson [2] that the speed of strictly dyadic $\mathrm{BBM}$ converges to $\sqrt{2 \beta}$ as $t \rightarrow \infty$ with probability one. On the other hand, Theorem $\mathrm{B}$ says that with probability one,

$$
\lim _{t \rightarrow \infty} \frac{\operatorname{vol}\left(Z_{t}^{r_{t}}\right)}{t}=2 \sqrt{2 \beta(1-k)} .
$$

Therefore, the large-time behavior of the volume of $\widehat{Z}_{t}^{r_{t}}$ is as different as it can be from that of $Z_{t}^{r_{t}}$. 


\section{Preparations}

\subsection{Preliminary results}

In this section, we develop preparatory results for the proofs of Theorem 2.1 and Theorem 2.2. The first result is about the large-time asymptotic probability of atypically large Brownian displacements. For a proof, see for example [9, Lemma 5]. As before, let $X=(X(t))_{t \geq 0}$ be a generic standard BM in $d$-dimensions, and $\mathbf{P}_{x}$ the law of $X$ started at position $x \in \mathbb{R}^{d}$, with corresponding expectation $\mathbf{E}_{x}$.

Proposition A (Linear Brownian displacements). For $\gamma>0$,

$$
\mathbf{P}_{0}\left(\sup _{0 \leq s \leq t}|X(s)|>\gamma t\right)=\exp \left[-\gamma^{2} t / 2+o(t)\right]
$$

The behavior as $t \rightarrow \infty$ of the expected volume of the Wiener sausage is well-known ([13], [8]):

$$
\mathbf{E}_{0}\left[\operatorname{vol}\left(X_{t}^{r}\right)\right]= \begin{cases}\sqrt{\frac{8 t}{\pi}}(1+o(1)), & d=1 \\ \frac{2 \pi t}{\log t}(1+o(1)), & d=2 \\ \kappa_{r} t(1+o(1)), & d \geq 3\end{cases}
$$

where $r>0$ is constant, and $\kappa_{r}=r^{d-2} 2 \pi^{d / 2} / \Gamma(d / 2-1)$ is the Newtonian capacity of $B(0, r)$ with $\Gamma$ denoting the gamma function. The expected volume of the shrinking Wiener sausage can be obtained from (3.1) and the scaling invariance of BM.

Lemma 3.1 (Expected volume of exponentially shrinking Wiener sausage). Let $k>0$, $r_{0}>0$, and $r: \mathbb{R}_{+} \rightarrow \mathbb{R}_{+}$be defined by $r(t)=r_{0} e^{-\beta k t}$. Then,

$$
\mathbf{E}_{0}\left[\operatorname{vol}\left(X_{t}^{r_{t}}\right)\right]= \begin{cases}\sqrt{\frac{8 t}{\pi}}(1+o(1)), & d=1, \\ \frac{\pi}{\beta k}(1+o(1)), & d=2, \\ \kappa_{r_{0}} t e^{-(d-2) \beta k t}(1+o(1)), & d \geq 3 .\end{cases}
$$

Proof. Write

$$
\bigcup_{0 \leq s \leq t} B\left(X(s), r_{0} e^{-\beta k t}\right)=e^{-\beta k t} \bigcup_{0 \leq s \leq t} B\left(X(s) e^{\beta k t}, r_{0}\right) .
$$

By scaling invariance, we have

$$
\mathbf{E}_{0}\left[\operatorname{vol}\left(\bigcup_{0 \leq s \leq t} B\left(X(s) e^{\beta k t}, r_{0}\right)\right)\right]=\mathbf{E}_{0}\left[\operatorname{vol}\left(\bigcup_{0 \leq s \leq t e^{2 \beta k t}} B\left(X(s), r_{0}\right)\right)\right] .
$$

Then, it follows from (3.3) and (3.4) that

$$
\mathbf{E}_{0}\left[\operatorname{vol}\left(\bigcup_{0 \leq s \leq t} B\left(X(s), r_{0} e^{-\beta k t}\right)\right)\right]=e^{-\beta(k d) t} \mathbf{E}_{0}\left[\operatorname{vol}\left(\bigcup_{0 \leq s \leq t e^{2 \beta k t}} B\left(X(s), r_{0}\right)\right)\right]
$$

Since $t e^{2 \beta k t} \rightarrow \infty$ as $t \rightarrow \infty$, it follows from (3.1) that

$$
\mathbf{E}_{0}\left[\operatorname{vol}\left(\bigcup_{0 \leq s \leq t e^{2 \beta k t}} B\left(X(s), r_{0}\right)\right)\right]= \begin{cases}\sqrt{\frac{8 t e^{2 \beta k t}}{\pi}}(1+o(1)), & d=1 \\ \frac{2 \pi t e^{2 \beta k t}}{\log \left(t e^{2 \beta k t}\right)}(1+o(1)), & d=2 \\ \kappa_{r_{0}} t e^{2 \beta k t}(1+o(1)), & d \geq 3\end{cases}
$$

Use (3.5) and (3.6) to complete the proof. 
Lemma 3.2 (Hitting probability of exponentially shrinking ball from outside). Let $k>0$, $r_{0}>0$, and $r: \mathbb{R}_{+} \rightarrow \mathbb{R}_{+}$be defined by $r(t)=r_{0} e^{-\beta k t}$. Fix $\rho \in \mathbb{R}^{d}$ such that $|\rho|=: R>r_{0}$. Then,

$$
\mathbf{P}_{\rho}\left(\min _{0 \leq s \leq 1}|X(s)|<r_{t}\right)=\frac{c_{R}}{\beta k t}(1+o(1)) \quad \text { in } d=2 \text {, }
$$

where $c_{R}=\int_{R^{2} / 2}^{\infty} \frac{e^{-x}}{2 x} d x$, and

$$
\mathbf{P}_{\rho}\left(\min _{0 \leq s \leq 1}|X(s)|<r_{t}\right)=c e^{-\beta k(d-2) t}(1+o(1)) \quad \text { in } d \geq 3,
$$

where $c=c\left(d, r_{0}, R\right)>0$.

Proof. For $d=2$, from [12, Eq. (1.6)] we have

$$
\lim _{r \rightarrow 0} \log \left(\frac{1}{r}\right) \mathbf{P}_{\rho}\left(\min _{0 \leq s \leq t}|X(s)|<r\right)=\int_{R^{2} /(2 t)}^{\infty} \frac{e^{-x}}{2 x} d x .
$$

Set $t=1$, and then replace $r$ by $r_{t}$ in the formula above. Then, (3.7) follows since $r_{t} \rightarrow 0$ as $t \rightarrow \infty$.

For $d \geq 3$, we follow an argument similar to the one in [12], which was developed for the case $d=2$. Recall that $R=|\rho|$ is fixed, and define $G$ and its Laplace transform $\widehat{G}$ for $\lambda>0$, respectively, as

$$
G(r, t ; R)=\mathbf{P}_{\rho}\left(\min _{0 \leq s \leq t}|X(s)|<r\right)=\mathbf{P}_{\rho}\left(\tau_{r} \leq t\right), \quad \widehat{G}(r, \lambda ; R)=\int_{0}^{\infty} e^{-\lambda t} G(r, t ; R) d t,
$$

where $\tau_{r}$ denotes the first hitting time of the Brownian motion to $B(0, r)$. It follows easily from Fubini's theorem that $\widehat{G}(r, \lambda ; R)=\frac{1}{\lambda} \mathbf{E}_{\rho}\left[e^{-\lambda \tau_{r}}\right]$. Then, from [6, Eq. (2.5)],

$$
\widehat{G}(r, \lambda ; R)=\frac{1}{\lambda}\left(\frac{r}{R}\right)^{v} \frac{K_{v}(\sqrt{2 \lambda} R)}{K_{v}(\sqrt{2 \lambda} r)},
$$

where $v=(d-2) / 2$, and $K_{v}$ is the modified Bessel function of the second kind of order $v$. Furthermore, for $d \geq 3$ (i.e., $v>0$ ), according to [6, Eq. (3.13)] and references therein,

$$
K_{v}(z)=\frac{\Gamma(v)}{2}\left(\frac{2}{z}\right)^{v}(1+o(1)), \quad z \rightarrow 0 .
$$

Then, it follows from (3.9) and (3.10) that

$$
\lim _{r \rightarrow 0} \frac{\widehat{G}(r, \lambda ; R)}{r^{2 v}}=\frac{2^{1-v / 2}}{\Gamma(v) R^{v}} \lambda^{v / 2-1} K_{v}(\sqrt{2 \lambda} R)=: \widehat{F}(\lambda) .
$$

An integral formula for $K_{v}(z)$ (see for example [6, p. 5240]) is

$$
K_{v}(z)=\frac{1}{2}\left(\frac{z}{2}\right)^{v} \int_{0}^{\infty} e^{-t-\frac{z^{2}}{4 t}} t^{-v-1} d t
$$

For $t \geq 0$, define $F(t)=\frac{1}{2^{v} \Gamma(v)} \int_{0}^{t} \frac{e^{-\frac{R^{2}}{2 x}}}{x^{1+v}} d x$. Then, using (3.12), it can be verified that

$$
\mathcal{L}[F(t)](\lambda)=\widehat{F}(\lambda)
$$

where $\mathcal{L}$ denotes the Laplace transform. For $r>0$, let $f_{r}$ be the probability density function for $\tau_{r}$, and define the measure $\mu_{r}(d t)=\frac{f_{r}(t)}{r^{2 v}} d t$ on $[0, \infty)$. Then, since $\widehat{G}(r, \lambda ; R)=$ $\frac{1}{\lambda} \mathbf{E}_{\rho}\left[e^{-\lambda \tau_{r}}\right]$, (3.11) is equivalent to

$$
\lim _{r \rightarrow 0} \mathcal{L}\left[\mu_{r}\right](\lambda)=\lim _{r \rightarrow 0} \int_{0}^{\infty} e^{-\lambda t} \mu_{r}(d t)=\lambda \widehat{F}(\lambda) .
$$


Due to (3.13), $\mathcal{L}\left[F^{\prime}(t)\right](\lambda)=\lambda \widehat{F}(\lambda)-F(0)$, and we have $F(0)=0$. It follows from (3.14) that

$$
\lim _{r \rightarrow 0} \int_{0}^{\infty} e^{-\lambda t} \mu_{r}(d t)=\int_{0}^{\infty} e^{-\lambda t} \mu(d t)
$$

where $\mu(d t)=F^{\prime}(t) d t$ on $[0, \infty)$. Then, by the continuity theorem for Laplace transforms, (3.15) implies that $\lim _{r \rightarrow 0} \mu_{r}([0, t])=\mu([0, t])$ for $t>0$, which is equivalent to

$$
\lim _{r \rightarrow 0} \frac{G(r, t ; R)}{r^{2 v}}=\frac{1}{2^{v} \Gamma(v)} \int_{0}^{t} \frac{e^{-\frac{R^{2}}{2 x}}}{x^{1+v}} d x .
$$

Setting $t=1$, and replacing $r$ by $r_{t}$ in (3.16) completes the proof of (3.8) since $r_{t} \rightarrow 0$ as $t \rightarrow \infty$.

\subsection{Heuristics}

It is clear that since the largest particle distance from the origin is $\sqrt{2 \beta} t+o(t)$ in a BBM, typically the volume of the BBM-sausage even with constant radius at time $t$ is not larger than $(\sqrt{2 \beta})^{d} \omega_{d}$ on the scale of $t^{d}$. On the other hand, we know from Lemma 3.1 that in $d=2$, the expected volume of the $r_{t}$-shrinking Wiener sausage is asymptotically constant. Therefore, since there are typically $e^{\beta t+o(t)}$ particles at time $t$, of which at least $e^{\varepsilon t}$ for some $\varepsilon>0$ can be treated as independent particles over the second half of the interval $[0, t]$, and since the volume of a subcritical ball grows only polynomially in $t$, we expect that in $d=2$ for large $t$ the $r_{t}$-shrinking BBM-sausage covers $B_{\theta}:=B(0, \theta \sqrt{2 \beta} t)$ for each $0<\theta<1$ provided that the particles of BBM spread out sufficiently homogeneously over $B_{\theta}$ (see Theorem A).

The situation is different in $d \geq 3$. The number of particles present at time $t$ with ancestral lines that have exited $B_{\theta}$ at some point over $[0, t]$ typically grows like $\exp [\beta t(1-$ $\left.\left.\theta^{2}\right)+o(t)\right]$. By the strong Markov property, such an ancestral line follows a typical Brownian path after it first exits $B_{\theta}$, and then stays out of $B_{\theta}$ over periods of total length at most $t$. Therefore, since the expected volume of an $r_{t}$-shrinking Wiener sausage decays like $\exp [-\beta k(d-2) t+o(t)]$ by Lemma 3.1, even if we suppose, for an upper bound on the volume of the BBM-sausage, that the aforementioned ancestral lines follow independent BMs starting from their first exit times out of $B_{\theta}$ up to $t$, and that over these time periods their respective $r_{t}$-shrinking Wiener sausages are all disjoint from one another, typically their union could only have a non-trivial volume (on the scale of $t^{d}$ ) outside $B_{\theta}$ provided that $1-\theta^{2} \geq k(d-2)$, which is equivalent to $\theta \leq \sqrt{1-k(d-2)}$. This explains the upper bound in (2.1). For the lower bound, consider a ball of unit size, say $B$, contained in $B_{\theta}:=B(0, \theta \sqrt{2 \beta} t)$ at time $t$, where $\theta<\sqrt{1-k(d-2)}$. Typically, the mass in $B$ at time $t-1$ is at least $\exp \left[\beta t\left(1-\theta^{2}\right)+o(t)\right]$. Also, due to (3.2), the expected volume scooped out over $[t-1, t]$ by the $r_{t}$-shrinking Wiener sausage is roughly $\mathbf{E}_{0}\left[\operatorname{vol}\left(X_{t}^{r_{t}}\right)\right] / t=\exp [-\beta t k(d-2)+o(t)]$. Then, provided that the particles are spread out sufficiently homogeneously over $B$, since $\theta<\sqrt{1-k(d-2)}$ and the volume of $B$ is constant, we expect even the $r_{t}$-shrinking Wiener sausages initiated by the particles present in $B$ at time $t-1$ to cover $B$ over $[t-1, t]$. Polynomially many balls of unit size suffice to cover $B_{\theta}$, and one can see with further analysis that a suitable union bound over these balls does not disturb the argument.

\section{Proof of main results}

We first give elementary bounds on $\operatorname{vol}\left(\widehat{Z}_{t}^{r_{t}}\right)$ that are valid in any dimension. Since $Z_{t}^{r_{t}} \subseteq \widehat{Z}_{t}^{r_{t}}$ by definition, it is clear that vol $\left(Z_{t}^{r_{t}}\right) \leq \operatorname{vol}\left(\widehat{Z}_{t}^{r_{t}}\right)$, and therefore, Theorem B 
implies that with probability one,

$$
\liminf _{t \rightarrow \infty} \frac{\operatorname{vol}\left(\widehat{Z}_{t}^{r_{t}}\right)}{t^{d}} \geq[2 \beta(1-k d)]^{d / 2} \omega_{d} .
$$

In the rest of the manuscript, let $\mathcal{N}_{t}$ denote the set of particles of $Z$ that are alive at time $t$, and set $N_{t}=\left|\mathcal{N}_{t}\right|$. For $u \in \mathcal{N}_{t}$, let $\left(Y_{u}(s)\right)_{0 \leq s \leq t}$ denote the ancestral line up to $t$ of particle $u$. By the ancestral line up to $t$ of a particle present at time $t$, we mean the continuous trajectory traversed up to $t$ by the particle, concatenated with the trajectories of all its ancestors including the one traversed by the initial particle. Note that $\left(Y_{u}(s)\right)_{0 \leq s \leq t}$ is identically distributed as a Brownian trajectory $(X(s))_{0 \leq s \leq t}$ for each $u \in \mathcal{N}_{t}$. Recall the definition of $R(t)$ from (1.1). For $t>0$, let $M_{t}:=\inf \{r \geq 0: R(t) \subseteq B(0, r)\}$. Then, using the union bound, for $\gamma>0$,

$$
P\left(M_{t}>\gamma t\right)=P\left(\exists u \in \mathcal{N}_{t}: \sup _{0 \leq s \leq t}\left|Y_{u}(s)\right|>\gamma t\right) \leq E\left[N_{t}\right] \mathbf{P}_{0}\left(\sup _{0 \leq s \leq t}|X(s)|>\gamma t\right) .
$$

It is a standard result that $E\left[N_{t}\right]=\exp (\beta t)$ (see for example [7, Sect. 8.11]). Moreover, we know from Proposition A that $\mathbf{P}_{0}\left(\sup _{0 \leq s \leq t}|X(s)|>\gamma t\right)=\exp \left[-\gamma^{2} t / 2+o(t)\right]$. Then, for fixed $\varepsilon>0$, defining the events

$$
A_{k}:=\left\{\operatorname{vol}\left(\widehat{Z}_{k}^{r_{k}}\right) / k^{d}>[2 \beta(1+\varepsilon)]^{d / 2} \omega_{d}\right\},
$$

it follows from setting $\gamma=\sqrt{2 \beta(1+\varepsilon)}$ in (4.2) that there exists a positive constant $c(\varepsilon)$ such that $P\left(A_{k}\right) \leq e^{-\beta c(\varepsilon) k}$ for all large $k$. Applying Borel-Cantelli lemma on the events $\left(A_{k}: k \geq 1\right)$, and then choosing $\varepsilon=1 / n$, and finally letting $n$ vary over $\mathbb{N}$ yields: with probability one,

$$
\limsup _{t \rightarrow \infty} \frac{\operatorname{vol}\left(\widehat{Z}_{t}^{r_{t}}\right)}{t^{d}} \leq(2 \beta)^{d / 2} \omega_{d}
$$

The proofs below 'close the gap' between $[2 \beta(1-k d)]^{d / 2} \omega_{d}$ in (4.1) and $(2 \beta)^{d / 2} \omega_{d}$ in (4.3) for $d=2$ and $d \geq 3$, separately. When $k=0$, observe that the lower bound in (4.1) coincides with the upper bound in (4.3), so there is nothing more to prove. Hence, in what follows, we suppose that $k>0$.

Definition 4.1 (Overwhelming probability). Let $\left(A_{t}\right)_{t>0}$ be a family of events indexed by time $t$. We say that $A_{t}$ occurs with overwhelming probability if there is a constant $k>0$ and time $t_{0}$ such that

$$
P\left(A_{t}^{c}\right) \leq e^{-k t} \text { for all } t \geq t_{0},
$$

where $A^{c}$ denotes the complement of event $A$.

\subsection{Proof of Theorem 2.1}

Note that $\omega_{2}=\pi$. The upper bound comes from (4.3). We will show that for every $\varepsilon>0$ there exists a positive constant $c_{1}$ such that for all large $t$,

$$
P\left(\operatorname{vol}\left(\widehat{Z}_{t}^{r_{t}}\right) / t^{2} \leq 2 \pi \beta(1-\varepsilon)\right) \leq e^{-c_{1} t} .
$$

Then, the lower bound for Theorem 2.1 will follow from (4.4) via a standard Borel-Cantelli argument.

Let $\varepsilon>0$, and for $t>0$ let $\rho_{t}:=\sqrt{2 \beta(1-\varepsilon)} t$ and $\mathbf{B}_{t}:=B\left(0, \rho_{t}\right)$. To prove (4.4), we choose a well-spaced net of points in $\mathbf{B}_{t}$, and argue that for large $t$ with overwhelming probability, each ball of radius one centered at a net point has sufficiently many particles at time $t-1$ so that even simple BMs initiated (rather than sub-BBMs) from the positions of these particles at time $t-1$ are enough to ensure that there is no ball of radius $r_{t}$ 
with center lying in $\mathbf{B}_{t}$ that remains not hit over the period $[t-1, t]$. In other words, the following occurs with overwhelming probability: over $[0, t-1]$, the system produces sufficiently many particles which are sufficiently well-spaced over $\mathbf{B}_{t}$ at time $t-1$, and then (neglecting the branching over $[t-1, t]$ ), Wiener sausages initiated from the positions of the particles at this time are enough to cover $\mathbf{B}_{t}$.

In this subsection, $d=2$. However, in some of the notation and arguments that follow, we prefer to keep $d$ general as they will be used in the next subsection as well, where $d \geq 3$.

For $t>0$ define

$$
m_{t}:=\left\lceil\frac{\rho_{t}}{1 /(2 \sqrt{d})}\right]^{d}, \quad n_{t}:=\left\lceil\frac{\rho_{t}}{r_{t} /(2 \sqrt{d})}\right]^{d} .
$$

Then, $n_{t}=\left\lceil c_{2} t / r_{t}\right\rceil^{d}$ for some $c_{2}=c_{2}(\varepsilon, \beta, d)$. For $t>0$, define the events

$$
A_{t}:=\left\{\operatorname{vol}\left(\widehat{Z}_{t}^{r_{t}}\right) / t^{2} \leq 2 \pi \beta(1-\varepsilon)\right\} .
$$

First, we prepare the setting at time $t-1$. Let $C\left(0, \rho_{t}\right)$ be the cube centered at the origin with side length $2 \rho_{t}$ so that $B\left(0, \rho_{t}\right)$ is inscribed in $C\left(0, \rho_{t}\right)$. Consider the simple cubic packing of $C\left(0, \rho_{t}\right)$ with balls of radius $1 /(2 \sqrt{d})$. Then, at most $m_{t}$ balls are needed to completely pack $C\left(0, \rho_{t}\right)$, say with centers $\left(x_{j}: 1 \leq j \leq m_{t}\right)$. For each $j$, let $B_{j}=B\left(x_{j}, 1 /(2 \sqrt{d})\right)$. (We suppress the $t$-dependence in $x_{j}$ and $B_{j}$ for ease of notation.) Consider a simple cubic packing of $\mathbb{R}^{d}$ by balls $\left(\mathcal{B}_{j}: j \in \mathbb{Z}_{+}\right)$of radius $r>0$, and let $x \in \mathbb{R}^{d}$ be any point. Then, it is easy to see that $\min _{j} \max _{z \in \mathcal{B}_{j}}|x-z|<(\sqrt{d} / 2) 4 r$, where $\sqrt{d} / 2$ is the distance between the center and any vertex of the $d$-dimensional unit cube, i.e., $C(0,1 / 2)$. Then, since the packing ball radius is $1 /(2 \sqrt{d})$ in our case, it follows that

$$
\forall x \in \mathbf{B}_{t}, \quad \min _{1 \leq j \leq m_{t}} \max _{z \in B_{j}}|x-z|<1 .
$$

For a Borel set $B \subseteq \mathbb{R}^{d}$ and $t \geq 0$, we write $Z_{t}(B)$ to denote the number of particles, i.e., the mass, of $Z$ that fall inside $B$ at time $t$. For $j \in\left\{1,2, \ldots, m_{t}\right\}$, define the events

$$
E_{j}:=\left\{Z_{t-1}\left(B_{j}\right)<e^{\beta(\varepsilon / 2) t}\right\} .
$$

Typically, the mass of BBM that fall inside a linearly moving ball of fixed radius $a>0$, say $B_{t}:=B(\theta \sqrt{2 \beta} t \mathbf{e}, a)$ for some unit vector e and $0<\theta<1$, is $\exp \left[\beta\left(1-\theta^{2}\right) t+o(t)\right]$. Quoting [10, Thm. 1], in any dimension $d \geq 1$, for $0 \leq a<1-\theta^{2}$,

$$
\lim _{t \rightarrow \infty} \frac{1}{t} \log P\left(Z_{t}\left(B_{t}\right)<e^{\beta a t}\right)=-\beta \times I
$$

for some positive rate function $I=I(\theta, a)$. Then, since $x_{j} \in B(0, \sqrt{2 \beta(1-\varepsilon)} t)$ for each $j$, $\beta \varepsilon / 2$ is an atypically small exponent (typical exponent is at least $\beta\left[1-(\sqrt{1-\varepsilon})^{2}\right]=\beta \varepsilon$ ) for the mass of BBM in each $B_{j}$ at time $t-1$. It follows from (4.8) that there exists a positive constant $c(\varepsilon)$ such that for all large $t$,

$$
P\left(\cup_{1 \leq j \leq m_{t}} E_{j}\right) \leq m_{t} e^{-c(\varepsilon) t}=e^{-c(\varepsilon) t+o(t)},
$$

where we have used the union bound and that $m_{t}$ is only a polynomial factor in $t$. It follows from (4.6), (4.7) and (4.9) that at time $t-1$, with overwhelming probability, there are at least $e^{\beta(\varepsilon / 2) t}$ particles in the 1-neighborhood of each point in $\mathbf{B}_{t}$. That is, there exists $c=c(\varepsilon)>0$ such that for all large $t$,

$$
P\left(G_{t}\right) \leq e^{-c t}, \quad G_{t}:=\left\{\inf _{x \in \mathbf{B}_{t}} Z_{t-1}(B(x, 1))<e^{\beta(\varepsilon / 2) t}\right\} .
$$


Now consider the simple cubic packing of $C\left(0, \rho_{t}\right)$ with balls of radius $r_{t} /(2 \sqrt{d})$. Then, at most $n_{t}$ balls are needed to completely pack $C\left(0, \rho_{t}\right)$, say with centers $\left(y_{j}: 1 \leq j \leq n_{t}\right)$. For each $j$, let $\widehat{B}_{j}=B\left(y_{j}, r_{t} /(2 \sqrt{d})\right)$. By an argument similar to the one leading to (4.6), it follows that

$$
\forall x \in \mathbf{B}_{t}, \quad \min _{1 \leq j \leq n_{t}} \max _{z \in \widehat{B}_{j}}|x-z|<r_{t} .
$$

For $j \in\left\{1,2, \ldots, n_{t}\right\}$, define the events $F_{j}:=\left\{Z_{s}\left(\widehat{B}_{j}\right)=0 \forall s \in[t-1, t]\right\}$. For $0 \leq t_{1} \leq t_{2}$, let

$$
R\left(t_{1}, t_{2}\right):=\bigcup_{s=t_{1}}^{t_{2}} \operatorname{supp}(Z(s)), \quad \widehat{Z}_{\left[t_{1}, t_{2}\right]}^{r}:=\bigcup_{x \in R\left(t_{1}, t_{2}\right)} B(x, r),
$$

that is, $R\left(t_{1}, t_{2}\right)$ is the accumulated support of $Z$ over $\left[t_{1}, t_{2}\right]$, and $\widehat{Z}_{\left[t_{1}, t_{2}\right]}^{r}$ is the corresponding sausage with radius $r$. For $t>0$, define the events

$$
H_{t}:=\left\{\exists x \in \mathbf{B}_{t} \text { such that } x \notin \widehat{Z}_{[t-1, t]}^{r_{t}}\right\} .
$$

It then follows from (4.11) that $H_{t} \subseteq \bigcup_{1 \leq j \leq n_{t}} F_{j}$, and therefore $P\left(H_{t} \mid G_{t}^{c}\right) \leq P\left(\cup_{1 \leq j \leq n_{t}} F_{j} \mid\right.$ $\left.G_{t}^{c}\right)$. Now, the union bound gives

$$
P\left(H_{t} \mid G_{t}^{c}\right) \leq n_{t} \max _{1 \leq j \leq n_{t}} P\left(F_{j} \mid G_{t}^{c}\right) .
$$

In view of (4.10), (4.12), and the estimate

$$
P\left(A_{t}\right) \leq P\left(H_{t}\right) \leq P\left(H_{t} \mid G_{t}^{c}\right)+P\left(G_{t}\right),
$$

and since $n_{t}$ is only an exponential factor in $t$, to complete the proof of (4.4), it suffices to show that $\max _{1 \leq j \leq n_{t}} P\left(F_{j} \mid G_{t}^{c}\right)$ is super-exponentially small in $t$ for large $t$.

Observe that conditional on the event $G_{t}^{c}$, the event $F_{j}$ for any $j$ can be realized only if the sub-BBMs initiated by each of the at least $\exp [\beta(\varepsilon / 2) t]$ many particles present in $B\left(y_{j}, 1\right)$ at time $t-1$ does not hit $B\left(y_{j}, r_{t} /(2 \sqrt{d})\right)$ in the remaining time interval $[t-1, t]$. Apply the Markov property at time $t-1$, and neglect possible branching of particles over $[t-1, t]$ for an upper bound on $P\left(F_{j} \mid G_{t}^{c}\right)$. Then, by (3.7) in Lemma 3.2, and the independence of particles present at time $t-1$, there exists $c>0$ such that for all large $t$,

$$
P\left(F_{j} \mid G_{t}^{c}\right) \leq\left(1-\mathbf{P}_{\mathbf{e}}\left(\min _{0 \leq s \leq 1}|X(s)|<\frac{r_{t}}{2 \sqrt{d}}\right)\right)^{e^{\beta(\varepsilon / 2) t}} \leq\left(1-\frac{c}{\beta k t}\right)^{e^{\beta(\varepsilon / 2) t}},
$$

where $\mathbf{e}$ is a unit vector. It is clear that the right-hand side of (4.13) is super-exponentially small in $t$ for large $t$. This completes the proof of Theorem 2.1.

\subsection{Proof of Theorem 2.2}

We will show that for every $\varepsilon>0$ there exist positive constants $c_{1}$ and $c_{2}$ such that for all large $t$,

$$
P\left(\operatorname{vol}\left(\widehat{Z}_{t}^{r_{t}}\right) / t^{d} \leq[2 \beta(1-k(d-2)-\varepsilon)]^{d / 2} \omega_{d}\right) \leq e^{-c_{1} t}
$$

and

$$
P\left(\operatorname{vol}\left(\widehat{Z}_{t}^{r_{t}}\right) / t^{d} \geq[2 \beta(1-k(d-2)+\varepsilon)]^{d / 2} \omega_{d}\right) \leq e^{-c_{2} t} .
$$

Then, Theorem 2.2 will follow from (4.14) and (4.15) via a standard Borel-Cantelli argument. 
The method of proof of (4.14) is identical to that of (4.4). We only need to make the following changes. For $t>0$, let $\rho_{t}:=\sqrt{2 \beta(1-k(d-2)-\varepsilon)} t$ and $\mathbf{B}_{t}:=B\left(0, \rho_{t}\right)$. Recall $m_{t}$ from (4.5), and that $B_{j}=B\left(x_{j}, 1 /(2 \sqrt{d})\right)$, and define the events $\left(A_{t}: t \geq 0\right)$ and $\left(E_{j}: j=1,2, \ldots, m_{t}\right)$ as

$$
A_{t}:=\left\{\operatorname{vol}\left(\widehat{Z}_{t}^{r_{t}}\right) / t^{d} \leq[2 \beta(1-k(d-2)-\varepsilon)]^{d / 2} \omega_{d}\right\}, \quad E_{j}:=\left\{Z_{t-1}\left(B_{j}\right)<e^{\beta(k(d-2)+\varepsilon / 2) t}\right\} .
$$

Then, since $\beta[k(d-2)+\varepsilon / 2]$ is an atypically small exponent (typical exponent is at least $\beta[k(d-2)+\varepsilon]$ ) for the mass in each $B_{j}$ at time $t-1$, by (4.8), there exists a positive constant $c(\varepsilon)$ such that for all large $t$,

$$
P\left(\cup_{1 \leq j \leq m_{t}} E_{j}\right) \leq m_{t} e^{-c(\varepsilon) t}=e^{-c(\varepsilon) t+o(t)} .
$$

The rest of the proof is identical to that of (4.4) except the last part, where we need to show that $P\left(F_{k} \mid G_{t}^{c}\right)$ is super-exponentially small in $t$ for large $t$. By (3.8) in Lemma 3.2, and the independence of particles present at time $t-1$, there exists $c>0$ such that for all large $t$,

$$
\begin{aligned}
P\left(F_{k} \mid G_{t}^{c}\right) & \leq\left(1-\mathbf{P}_{\mathbf{e}}\left(\min _{0 \leq s \leq 1}|X(s)|<\frac{r_{t}}{2 \sqrt{d}}\right)\right)^{e^{\beta(k(d-2)+\varepsilon / 2) t}} \\
& \leq\left(1-c e^{-\beta k(d-2) t}\right)^{e^{\beta(k(d-2)+\varepsilon / 2) t}} \cdot
\end{aligned}
$$

Using that $1+x \leq e^{x}$, the right-hand side of (4.16) can be bounded from above by $\exp \left[-c e^{\beta(\varepsilon / 2) t}\right]$, which is super-exponentially small in $t$ for large $t$. This completes the proof of (4.14).

To prove (4.15), for $0 \leq \theta<1$, let $B_{\theta}:=B(0, \theta \sqrt{2 \beta} t)$, and as before, for $u \in \mathcal{N}_{t}$, let $\left(Y_{u}(s)\right)_{0 \leq s \leq t}$ denote the ancestral line up to $t$ of particle $u$. Next, for $u \in \mathcal{N}_{t}$, let

$$
\tau_{u}=\inf \left\{s \geq 0:\left|Y_{u}(s)\right|>\theta \sqrt{2 \beta} t-r_{t}\right\},
$$

the first time the sausage associated with the ancestral line of particle $u$ exits $B_{\theta}$, and let

$$
\tau=\inf \left\{s \geq 0:|X(s)|>\theta \sqrt{2 \beta} t-r_{t}\right\}
$$

where $X=(X(s))_{s \geq 0}$ is a standard BM. Let $\widehat{Z}_{t}^{r_{t}, \theta}$ be the part of the sausage $\widehat{Z}_{t}^{r_{t}}$ outside $B_{\theta}$, that is, $\widehat{Z}_{t}^{r_{t}, \theta}:=\widehat{Z}_{t}^{r_{t}} \cap B_{\theta}^{c}$, where $B_{\theta}^{c}$ denotes the complement of $B_{\theta}$ in $\mathbb{R}^{d}$. Then,

$$
\begin{aligned}
E\left[\operatorname{vol}\left(\widehat{Z}_{t}^{r_{t}, \theta}\right)\right] & \leq E\left[\sum_{u \in \mathcal{N}_{t}} \mathbb{1}_{\left\{\tau_{u}<t\right\}} \cdot \operatorname{vol}\left(\cup_{\tau_{u} \leq s \leq t} B\left(Y_{u}(s), r_{t}\right)\right)\right] \\
& =E\left[N_{t}\right] \mathbf{E}_{0}\left[\mathbb{1}_{\{\tau<t\}} \cdot \operatorname{vol}\left(\cup_{\tau \leq s \leq t} B\left(X(s), r_{t}\right)\right)\right]
\end{aligned}
$$

by the many-to-one lemma, where $\mathbb{1}_{A}$ denotes the indicator function of $A$. By conditioning on $\mathcal{F}_{\tau}$, the $\sigma$-algebra associated with the stopping time $\tau$, and then using the strong Markov property of BM,

$$
\begin{aligned}
\mathbf{E}_{0}\left[\mathbb{1}_{\{\tau<t\}} \cdot \operatorname{vol}\left(\cup_{\tau \leq s \leq t} B\left(X(s), r_{t}\right)\right)\right] & =\mathbf{E}_{0}\left[\mathbb{1}_{\{\tau<t\}} \mathbf{E}_{0}\left[\operatorname{vol}\left(\cup_{\tau \leq s \leq t} B\left(X(s), r_{t}\right)\right) \mid \mathcal{F}_{\tau}\right]\right] \\
& \leq \mathbf{E}_{0}\left[\mathbb{1}_{\{\tau<t\}} \mathbf{E}_{0}\left[\operatorname{vol}\left(\cup_{\tau \leq s \leq t+\tau} B\left(X(s), r_{t}\right)\right) \mid \mathcal{F}_{\tau}\right]\right] \\
& =\mathbf{E}_{0}\left[\mathbb{1}_{\{\tau<t\}} \mathbf{E}_{0}\left[\operatorname{vol}\left(X_{t}^{r_{t}}\right)\right]\right] \\
& =\mathbf{P}_{0}(\tau<t) \mathbf{E}_{0}\left[\operatorname{vol}\left(X_{t}^{r_{t}}\right)\right]
\end{aligned}
$$

Hence, by (4.17), Proposition A, and the fact that $E\left[N_{t}\right]=e^{\beta t}$,

$$
E\left[\operatorname{vol}\left(\widehat{Z}_{t}^{r_{t}, \theta}\right)\right] \leq e^{\beta t} e^{-\theta^{2} \beta t+o(t)} \mathbf{E}_{0}\left[\operatorname{vol}\left(X_{t}^{r_{t}}\right)\right]
$$


Then, by (4.19) and Lemma 3.1, we have

$$
E\left[\operatorname{vol}\left(\widehat{Z}_{t}^{r_{t}, \theta}\right)\right] \leq e^{\beta t\left(1-\theta^{2}-k(d-2)\right)+o(t)} .
$$

Let $\theta_{1}=\sqrt{1-k(d-2)+\varepsilon / 2}$. It follows from the Markov inequality and (4.20) that

$$
P\left(\operatorname{vol}\left(\widehat{Z}_{t}^{r_{t}, \theta_{1}}\right) \geq 1\right) \leq e^{-\beta \varepsilon t / 2+o(t)} .
$$

Observe that $\operatorname{vol}\left(\widehat{Z}_{t}^{r_{t}}\right) \leq \operatorname{vol}\left(\widehat{Z}_{t}^{r_{t}, \theta_{1}}\right)+\left(\theta_{1} \sqrt{2 \beta} t\right)^{d} \omega_{d}<[2 \beta(1-k(d-2)+\varepsilon)]^{d / 2} \omega_{d} t^{d}$ for all large $t$ conditional on the event $\left\{\operatorname{vol}\left(\widehat{Z}_{t}^{r_{t}, \theta_{1}}\right)<1\right\}$. This, along with (4.21), implies (4.15), and hence completes the proof of Theorem 2.2.

\section{References}

[1] Bolthausen, E.: On the volume of the Wiener sausage. Ann. Probab. 18(4), (1992), 1576 1582. MR-1071810

[2] Bramson, M.: Maximal displacement of branching Brownian motion. Comm. Pure Appl. Math. 31(5), (1978), 531 - 581. MR-0494541

[3] Donsker, M. D. and Varadhan, S. R. S.: Asymptotics for the Wiener sausage. Comm. Pure Appl. Math. 28(4), (1975), 525 - 565. MR-0397901

[4] Engländer, J.: On the volume of the supercritical super-Brownian sausage conditioned on survival. Stochastic Process. Appl. 88, (2000), 225 - 243. MR-1767846

[5] Hamana, Y.: On the expected volume of the Wiener sausage. J. Math Soc. Jpn. 62(4), (2010), 1113 - 1136. MR-2761916

[6] Hamana, Y. and Matsumoto, H.: The probability distributions of the first hitting times of Bessel processes. Trans. Amer. Math Soc. 365(10), (2013), 5237 - 5257. MR-3074372

[7] Karlin, S. and Taylor, M.: A First Course in Stochastic Processes. Second edition. Academic Press, New York-London, 1975. xvii+557 pp. MR-0356197

[8] Le Gall, J.-F.: Sur une conjecture de M. Kac. Probab. Theory Relat. Fields 78(3), (1988), 389 402. MR-0949180

[9] Öz, M., Çağlar, M. and Engländer, J.: Conditional speed of branching Brownian motion, skeleton decomposition and application to random obstacles. Ann. Inst. H. Poincaré Probab. Statis. 53(2), (2017), 842 - 864. MR-3634277

[10] Öz, M.: Large deviations for local mass of branching Brownian motion, arXiv:1811.09037

[11] Öz, M.: On the density of branching Brownian motion in subcritical balls, arXiv:1909.06197

[12] Spitzer, F.: Some theorems concerning 2-dimensional Brownian motion. Trans. Amer. Math. Soc. 87(1), (1958), 187 - 197. MR-0104296

[13] Spitzer, F.: Electrostatic Capacity, Heat Flow, and Brownian Motion. Z. Wahrsch. und Verw. Gebiete 3(2), (1964), 110 - 121. MR-0172343

[14] Sznitman, A.-S.: Long time asymptotics for the shrinking wiener sausage. Comm. Pure Appl. Math. 43(6), (1990), 809 - 820. MR-1059329

Acknowledgments. The author would like to thank the anonymous referee for pointing out a mistake in one of the proofs, and for helpful suggestions, including a simpler proof for one of the equations, that led to a significantly better presentation. 